\title{
Subendocardial Increase in Reactive Oxygen Species Production Affects Regional Contractile Function in Ischemic Heart Failure
}

\author{
Lucas Andre, ${ }^{1, \star}$ Jeremy Fauconnier, ${ }^{1, *}$ Cyril Reboul, ${ }^{2, *}$ Christine Feillet-Coudray, ${ }^{3}$ Pierre Meschin, \\ Charlotte Farah, ${ }^{2}$ Gilles Fouret, ${ }^{3}$ Sylvain Richard, ${ }^{1}$ Alain Lacampagne,, and Olivier Cazorla ${ }^{1}$
}

\begin{abstract}
Aims: Heart failure (HF) is characterized by regionalized contractile alterations resulting in loss of the transmural contractile gradient across the left ventricular free wall. We tested whether a regional alteration in mitochondrial oxidative metabolism during HF could affect myofilament function through protein kinase A (PKA) signaling. Results: Twelve weeks after permanent left coronary artery ligation that induced myocardial infarction (MI), subendocardial (Endo) cardiomyocytes had decreased activity of complex I and IV of the mitochondrial electron transport chain and produced twice more superoxide anions than sham Endo and subepicardial cells. This effect was associated with a reduced antioxidant activity of superoxide dismutase and Catalase only in MI Endo cells. The myofilament contractile properties $\left(\mathrm{Ca}^{2+}\right.$ sensitivity and maximal tension), evaluated in skinned cardiomyocytes, were also reduced only in MI Endo myocytes. Conversely, in MI rats treated with the antioxidant N-acetylcysteine (NAC) for 4 weeks, the generation of superoxide anions in Endo cardiomyocytes was normalized and the contractile properties of skinned cardiomyocytes restored. This effect was accompanied by improved in vivo contractility. The beneficial effects of NAC were mediated, at least, in part, through reduction of the PKA activity, which was higher in MI myofilaments, particularly, the PKA-mediated hyperphosphorylation of cardiac Troponin I. Innovation: The Transmural gradient in the mitochondrial content/activity is lost during HF and mediates reactive oxygen speciesdependent contractile dysfunction. Conclusions: Regionalized alterations in redox signaling affect the contractile machinery of sub-Endo myocytes through a PKA-dependent pathway that contributes to the loss of the transmural contractile gradient and impairs global contractility.
\end{abstract}

\section{Introduction}

U

NDER PHYSIOLOGICAL CONDITIONS, heart displays a transmural contractile gradient across the left ventricle (LV) due to higher contractility of the subendocardial (Endo) layer in comparison to the subepicardial (Epi) layer $(9,17)$. This gradient results from the transmural excitation-contraction coupling heterogeneity with longer action potential $(3,21)$, higher sarcoplasmic reticulum $\mathrm{Ca}^{2+}$ release (34), and higher myofilament $\mathrm{Ca}^{2+}$ sensitivity $(14,15)$ in the Endo than in the Epi layer. Excitation-contraction coupling requires vast amount of ATP and depends on the cardiac metabolic properties, which also show regional differences across the LV free wall. Indeed, the Endo layer is characterized by higher perfusion, oxygen consumption, and oxidative metabolism than the Epi layer $(19,28)$.
Heart failure (HF) is a syndrome characterized by the inability of the heart to provide a sufficient cardiac output due to

\section{Innovation}

Oxidative metabolism is altered during heart failure (HF). The present work shows the presence of a transmural gradient in the mitochondrial content/activity, which is lost during HF. This is due to alterations of the subendocardial layer, which mediates contractile dysfunction, at least, in part, by a protein kinase A-dependent pathway. Antioxidant supplementation may be considered as a complementary treatment to the current medical therapies, such as $\beta$-blockers, for patients with HF.

\footnotetext{
${ }^{1}$ U1046, INSERM, Université Montpellier 1, Université Montpellier 2, Montpellier, France.

${ }^{2}$ EA-4278, Université Avignon et des Pays de Vaucluse, UFR Sciences, Avignon, France.

${ }^{3}$ INRA UMR 866, Unité Différentiation Cellulaire et Croissance, Montpellier, France.

*These authors have equally contributed to the work.
} 
regional contractile defects that are mainly localized in the Endo layer $(15,34)$, leading to loss of the transmural contractile heterogeneity of the LV $(14,16)$. We showed previously in rat that moderate exercise started at a late stage of HF was able to restore a transmural contractile gradient by improving cellular contractility in the Endo layer $(14,34)$. It is not clear why exercise during HF has a cardio-protective effect, but increased antioxidant activity might play an important role (23). The failing heart displays altered mitochondrial function characterized by changes in the energy and oxidative metabolism (35) and abnormal reactive oxygen species (ROS) production (22). ROS can regulate proteins involved in excitation-contraction coupling (46), notably through activation of protein kinase A (PKA) and subsequent phosphorylation of PKA substrates (11). This might contribute to the HF-associated contractile defects (22). In addition, in vitro oxidation of myofilaments affects negatively the contractile machinery properties (24). However, it is not known whether alterations in ROS production are responsible for the regional contractile defects observed in HF.

Here, we investigated in a rat model 12 weeks after myocardial infarction (MI): (i) whether changes in transmural mitochondrial oxidative metabolism could participate in the development of the contractile machinery defects observed in ischemic HF through the redox/PKA pathway, and (ii) whether antioxidant treatment with $\mathrm{N}$-acetylcysteine (NAC) could improve the morphofunctional remodeling of single cardiomyocytes and of the whole heart (Fig. 1). The results indicate that HF-associated defects are predominantly localized in Endo cells, where increased ROS production during pacing affects negatively the contractile machinery through a PKA-dependent pathway. Chronic treatment with NAC improved the global cardiac function by restoring the contractile properties of myofilaments in rats with HF.

\section{Results}

Cardiomyocytes from $\mathrm{MI}$ rats show transmural differences in ROS production

Mitochondria are the main ATP provider in cardiomyocytes to match the energy demand and supply. We first in-

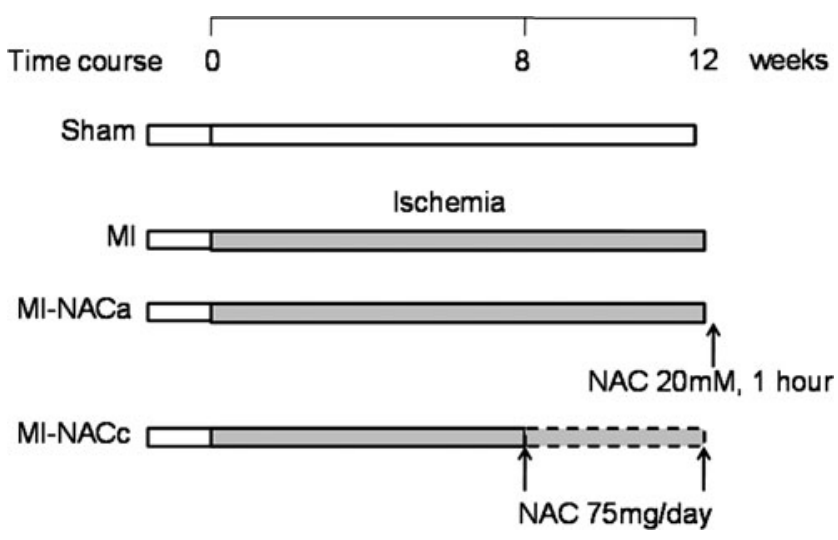

FIG. 1. Experimental design. The five experimental groups consisted of sham rats, untreated MI rats (MI), MI myocytes treated with $20 \mathrm{mM}$ NAC for $1 \mathrm{~h}$ (MI-NACa) and rats treated 2 months after infarction with NAC in the drinking water (75 mg/day) during 1 month (MI-NACc). MI, myocardial infarction; NAC, N-acetylcysteine. vestigated whether regional mitochondrial function/content was altered in MI rats. To this aim, we first assessed in the isolated cardiomyocytes citrate synthase (CS) activity, as it is considered as an index of mitochondrial oxidative metabolism (44) and/or of mitochondrial content (30). We also measured the activity of complex I and IV (normalized to CS activity), which oxidize, respectively, redox carriers NADH and cytochrome $\mathrm{c}$ in the inner mitochondrial membrane (38). In sham rats, the CS activity was significantly higher in Endo than in Epi cardiomyocytes. In MI rats, the CS activity was decreased only in Endo cells to levels comparable to those of Epi cells, suggesting a potential loss of the transmural gradient in the mitochondrial content/oxidative metabolism (Fig. 2A). Similarly, complex I and IV activities were significantly higher in Endo than in Epi cardiomyocytes from sham rats (Fig. 2A). Conversely, in Endo cells from MI rats, they were both decreased to the level observed in MI Epi cells, indicating a regional alteration of the mitochondrial respiratory chain following MI.

A deficient complex I activity during HF has been associated with increased ROS production (26). To test if during contraction MI Endo cells produce more ROS, we measured the mitochondrial production of superoxide anions $\left(\mathrm{O}_{2}{ }^{\bullet-}\right)$ in contracting intact Endo and Epi cardiomyocytes from sham and MI rats using MitoSOX Red, which increases its fluorescence when oxidized (2) (Fig. 2B). Although MitoSOX Red is less suitable for detecting differences in basal mitochondrial ROS production between different cell populations, it can readily detect changes in mitochondrial ROS production during an experiment (2). In these conditions in sham animals, the dynamic ROS production was comparable in both types of cells. Conversely, in MI rats, ROS generation increased significantly only in the Endo layer compared with Sham animals. The higher production of ROS in the Endo compared with the Epi layer ( $37 \% \pm 5 \%$ vs. $12 \% \pm 4 \%$ ) indicated the development of a transmural gradient in mitochondrial ROS production following MI (Fig. 2B). ROSinduced damages in the tissue were investigated by measuring the content of Malondialdehyde (MDA), an endproduct of lipid peroxidation, in the myocardial tissue isolated from the sub-Endo and sub-Epi of sham/MI/MINACc hearts mounted in the Langendorff apparatus and paced to $7 \mathrm{~Hz}$ for $15 \mathrm{~min}$ before rapid dissection. The MDA content was higher in Endo MI hearts compared with sham animals (Fig. 2C). As oxidative stress results from an imbalance between ROS production and antioxidant response, we evaluated the activity of two key antioxidant enzymes: superoxide dismutase (SOD), which converts $\mathrm{O}_{2}{ }^{-\bullet}$ to $\mathrm{H}_{2} \mathrm{O}_{2}$, and Catalase, which converts $\mathrm{H}_{2} \mathrm{O}_{2}$ to $\mathrm{H}_{2} \mathrm{O}$ (Fig. 2D). In sham rats, SOD and Catalase activities were higher in Endo than in Epi cardiomyocytes, whereas in MI rats their activity was reduced only in Endo cells.

These results suggest that, in HF, Endo cells have altered mitochondrial oxidative metabolism and produce more ROS during contraction. The ROS production and MDA content in Endo myocytes and tissue were normalized in MI rats treated for 4 weeks with the antioxidant NAC (MI-NACc) starting 8 weeks after MI (Fig. 2B, C). Similarly, chronic NAC treatment of MI rats normalized the activities of complexes I and IV and the antioxidant enzymes in the Endo cardiomyocytes to sham levels (Supplementary Fig. S2; Supplementary Data are available online at www.liebertpub.com/ars). 
FIG. 2. Regionalized impairment in mitochondrial activities during heart failure. (A) CS, complex I and complex IV activities in Endo and Epi layers of sham and MI hearts (eight hearts for each group). (B) Top panels: Typical images of mitochondrial $\mathrm{O}_{2}{ }^{\bullet-}$ production at rest and after pacing using MitoSOX Red in Endo cells from sham and MI rats. Bottom panel: Quantification of mitochondrial $\mathrm{O}_{2}{ }^{\bullet-}$ production after pacing in Endo and Epi cardiomyocytes isolated from sham (open bars), MI (dashed bars) and MI rats treated with NAC for 4 weeks (MI-NACc, gray bars) (32-40 cells $/ 4$ hearts/each condition). (C) MDA content in myocardial tissue isolated hearts that has been paced at $7 \mathrm{~Hz}$ for $15 \mathrm{~min}$ using Langendorff apparatus. (D) SOD and catalase activity (in $\mathrm{U} / \mathrm{mg}$ protein) in Endo and Epi layers of sham and MI hearts (eight hearts for each group). ${ }^{*} p<0.05 \mathrm{MI}$ versus sham within each layer, ${ }^{\dagger} p<0.05$ Endo versus Epi. Scale bar $=40 \mu \mathrm{m}$. Endo, endocardium; Epi, epicardium; $\mathrm{CS}$, citrate synthase; MDA, malondialdehyde; SOD, superoxide dismutase.
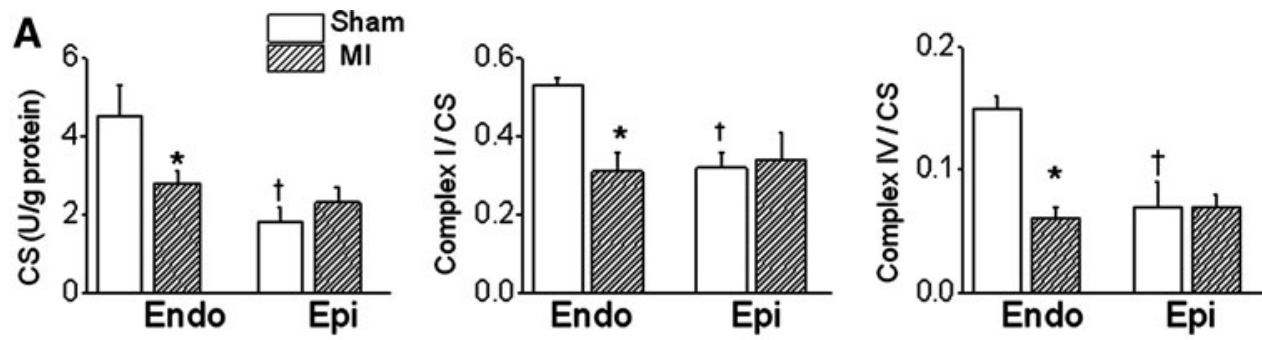
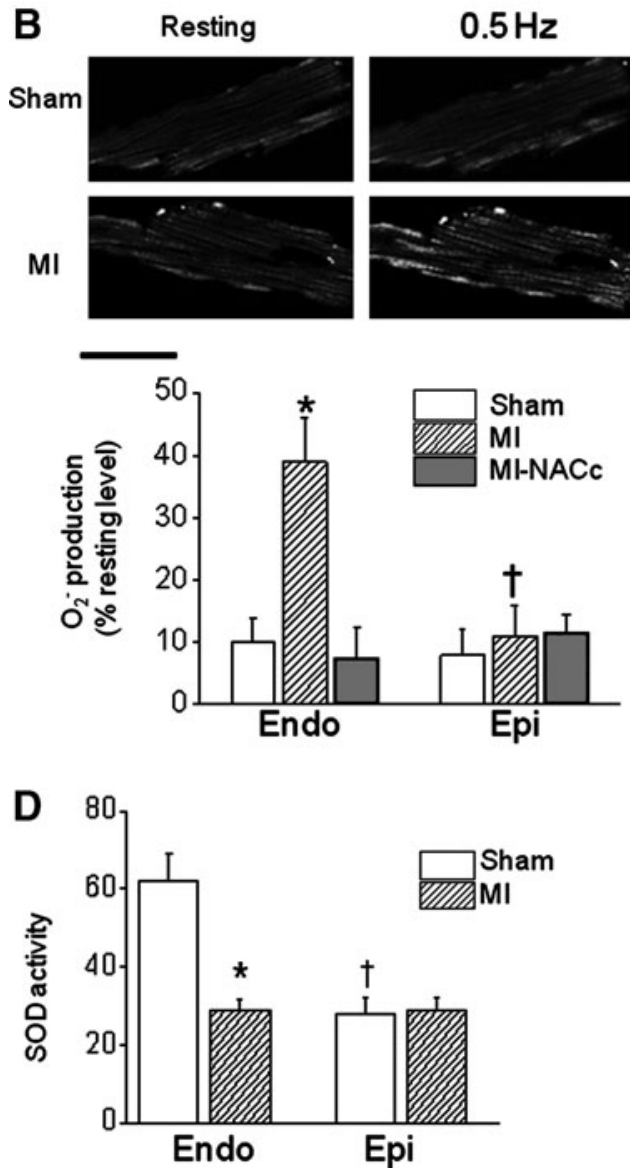

C
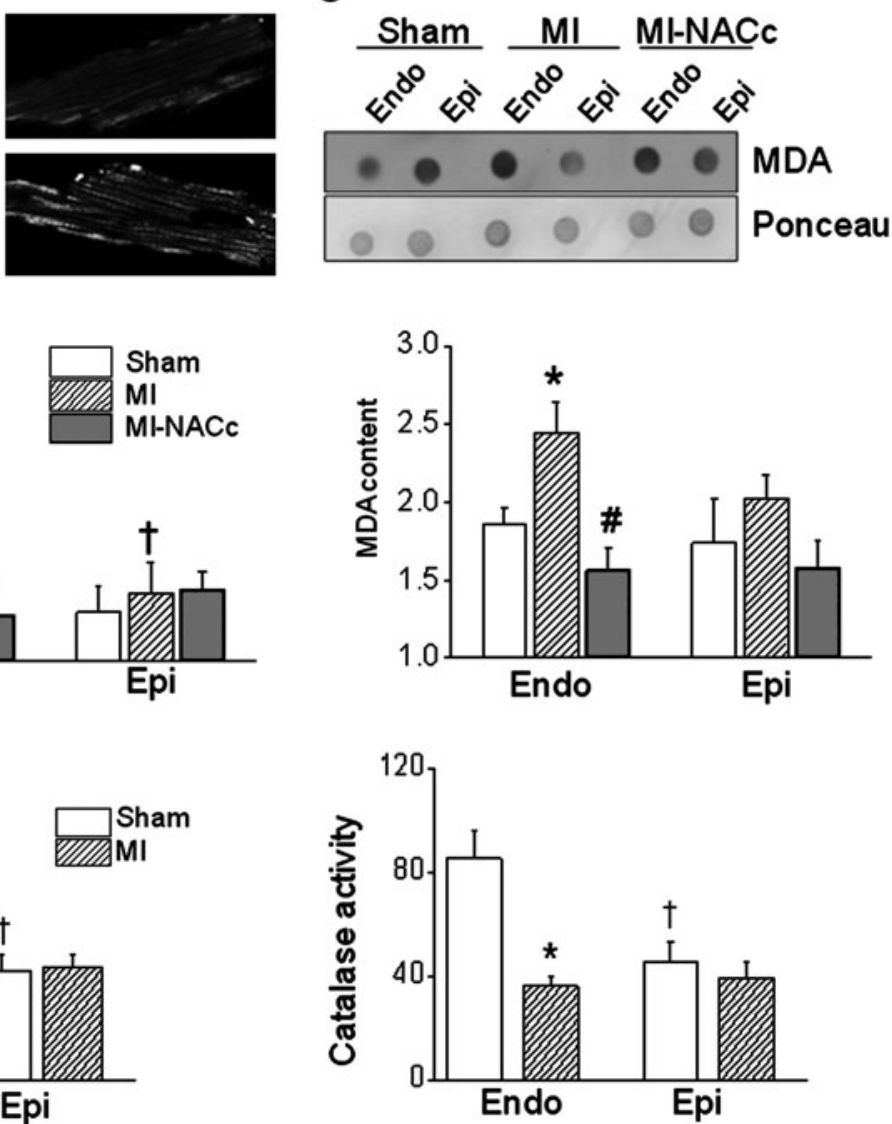

Effects of regionalized ROS production on the contractile machinery

Next, we evaluated the impact of ROS scavenging on the contractile machinery properties in MI myocytes. The relationship between the $\mathrm{Ca}^{2+}$-activated tension and the internal $\mathrm{Ca}^{2+}$ concentration was determined in single permeabilized cardiomyocytes (Fig. 3A and Supplementary Table S1). Force in MI Endo cardiomyocytes was reduced as indicated by the lower maximal active tension (Fig. 3B) and myofilament $\mathrm{Ca}^{2+}$ sensitivity (i.e., the $\mathrm{pCa}$ units producing one-half maximal tension, $\mathrm{pCa}_{50}$ ) in comparison to sham Endo cells (Fig. 3C). Acute treatment of intact MI myocytes with $20 \mathrm{mM}$ NAC for $1 \mathrm{~h}$ (MINACa cells) before permeabilization reversed the contractile abnormalities ( $\mathrm{pCa}_{50}$ and maximal active tension) observed in Endo cells. Epi cells were not affected by acute NAC treatment. Similarly, chronic NAC treatment (MI-NACc) prevented the reduction of contractility in Endo cells and even increased myofilament $\mathrm{Ca}^{2+}$ sensitivity in Epi cells to values higher than in sham Epi cells (Fig. 3C). These results suggest that the reduced contractility of MI Endo cardiomyocytes might be ROSdependent as it can be reversed by NAC treatment.

As in vitro oxidation of sulfhydryl groups of myofilament proteins reduces the contractile force and myofilament $\mathrm{Ca}^{2+}$ sensitivity in human control myocytes (24), we then assessed the occurrence of post-translational modifications of proteins in Endo and Epi from MI, MI-NACc, and sham rats using the Oxyblot kit (Fig. 4). The level of carbonylation was quantified for the total protein content and in isolated bands from 12 to $130 \mathrm{kDa}$, but no decrease in carbonylation was observed after chronic NAC treatment (Fig. 4B). Similarly, the level of carbonylation of immunoprecipitated cardiac Troponin I (cTnI) was unchanged in MI and MI-NACc rats in comparison to controls (Fig. 4C). Although carbonylation is the most common post-translational modification induced by ROS and severe oxidative stress, it could not be detected in our conditions. This might suggest that the effect of oxidative stress on myofilament function is indirect. 

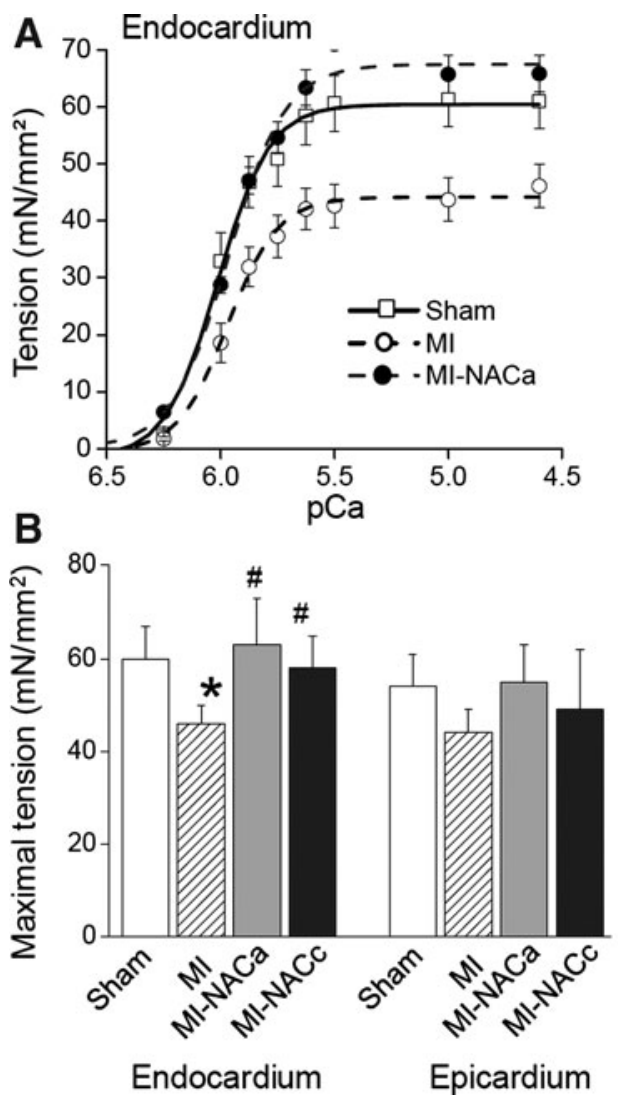

\section{ROS alterations of myofilaments in HF are PKA-mediated}

Myofilament $\mathrm{Ca}^{2+}$ desensitization in HF is usually associated with PKA-dependent phosphorylation of contractile proteins (29). We, thus, investigated the role of PKA in myofilament $\mathrm{Ca}^{2+}$ sensitivity by incubating myofilaments with a recombinant catalytic subunit of PKA. After PKA incubation, myofilament $\mathrm{Ca}^{2+}$ sensitivity decreased in sham and $\mathrm{MI}$ myocytes from both layers and differences between conditions disappeared (Fig. 5A). A similar decrease was observed with myofilaments obtained from intact MI myocytes preincubated with NAC for $1 \mathrm{~h}$ (MI-NACa) before permeabilization. This result suggests that the differences in $\mathrm{pCa}_{50}$ observed in sham and MI cells might be due to different levels of PKA activation and might be modulated by NAC treatment. The level of PKA activity measured directly in cardiomyocytes was significantly higher in the Endo (110\% increase) and Epi layers (33\%) from MI rats than from sham rats, in which it was comparable across the wall (Fig. 5B). Acute treatment with NAC normalized the level of PKA activity in both layers of MI hearts (MI-NACa) to the sham level. Moreover, chronic NAC treatment of MI rats (MI-NACc) further reduced the PKA activity in the Endo layer. Similarly, the phosphorylation level of cTnI, which is a major regulatory protein of myofilaments and a PKA target, was increased only in MI cardiomyocytes from the Endo layer (Fig. 5C). This increase was not observed in MI Endo cells after acute (MINACa) and chronic (MI-NACc) NAC treatment. The absence of hyperphosphorylation in MI Epi cardiomyocytes could be explained by the fact that the weak PKA activation in this layer was not sufficient to phosphorylate the target protein.
The activity of PKA is modulated by the level of cAMP. We have, thus, measured the concentration of cAMP in cardiomyocytes isolated from Sham, MI, and MI-NAC hearts. The level of cAMP did not differ between myocytes isolated from the Endo layer of Sham, MI, and MI-NAC hearts and, thus, cannot explain the higher PKA activity in Endo MI myocytes (Fig. 5D). To explore the potential activation of PKA by ROS and its effects on myofilament $\mathrm{Ca}^{2+}$ sensitivity, intact sham myocytes were incubated with $0.1 \mathrm{mM} \mathrm{H}_{2} \mathrm{O}_{2}$ for $15 \mathrm{~min}$ before permeabilization with TritonX100. The PKA activity (Fig. 5B) and the phosphorylation level of cTnI (Fig. 5E) increased and myofilament $\mathrm{Ca}^{2+}$ sensitivity decreased significantly (Fig. 5E) in control myocytes stimulated with $\mathrm{H}_{2} \mathrm{O}_{2}$ in comparison to nonstimulated cells. Coincubation with $2 \mu M$ KT5720, a specific PKA inhibitor, prevented the $\mathrm{H}_{2} \mathrm{O}_{2^{-}}$ dependent increase of cTnI phosphorylation and decrease of myofilament $\mathrm{Ca}^{2+}$ sensitivity. Incubation of permeabilized myocytes with $\mathrm{H}_{2} \mathrm{O}_{2}$ had no effect on the myofilament contractile properties, indicating that the effect of ROS is not direct on the myofilaments, but requires functional cytoplasmic membrane, sarcoplasmic reticulum, and mitochondria that are affected by the permeabilization procedure to activate signaling pathways.

Taken together, the results indicate that increased ROS production in MI Endo cardiomyocytes activates PKA, which then modulates the phosphorylation of proteins involved in myofilament regulation; treatment of failing cardiomyocytes and rats with an antioxidant prevents these alterations.

\section{Effects of antioxidant treatment on the contractile gradient in $\mathrm{MI}$ rats}

The impact of the ROS-dependent effects on the contractile machinery was next explored in intact myocytes. In MI rats, 
FIG. 4. Oxidation of sarcomeric proteins in LV myocardium. (A) Total proteins extracted from LV myocardium $(5 \mu \mathrm{g})$ of sham, MI, and MI-NAC rats were derivatized with DNP to detect protein carbonylation and were analyzed by immunoblotting with anti-DNP antibodies (left panel). cTnI was identified by immunoblotting on the same membrane (right panel). (B) Quantification of the oxidation index (i.e., the ratio between the densitometric values of total and single Oxyblot bands and that of the cTnI band $(n=6$ hearts per group). (C) cTnI was immunoprecipitated and its carbonylation level determined ( $n=3$ hearts). LV, left ventricle; MW, molecular weight.
A

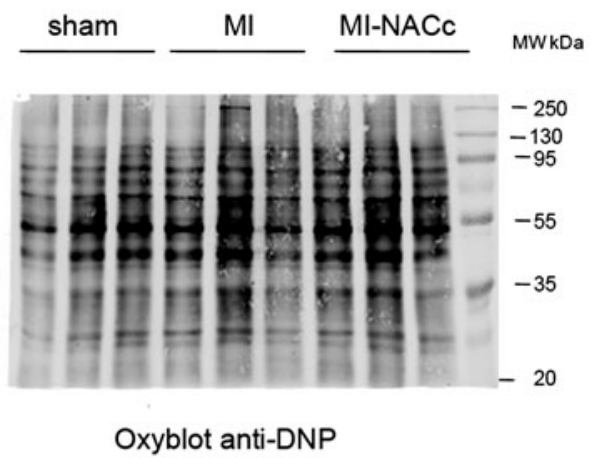

B
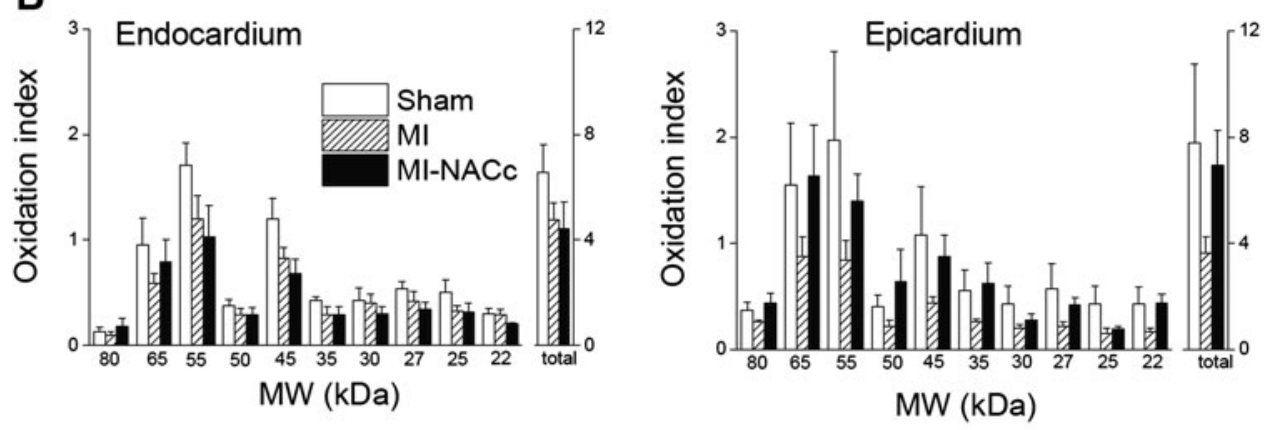

C

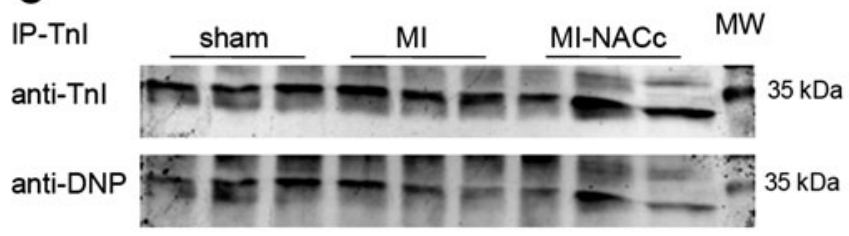

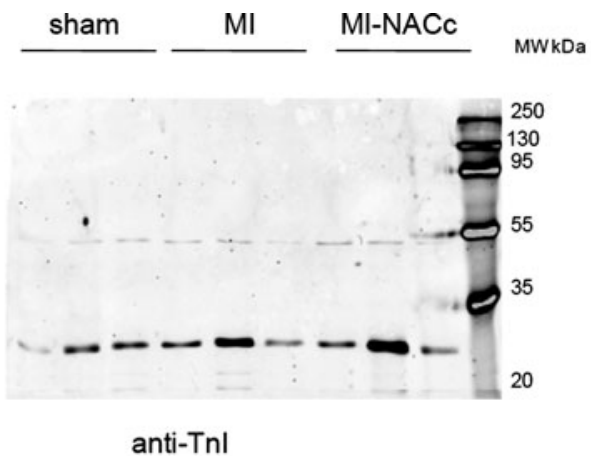

anti-Tnl
$\mathrm{MW}(\mathrm{kDa})$

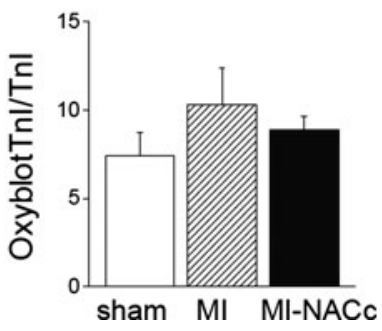

the transmural gradient of contractility observed in sham animals disappeared due to reduced sarcomere length (SL) shortening in the Endo layer (Fig. 6A, B). Moreover, both contraction and relaxation velocities were reduced in Endo myocytes from MI rats (Fig. 6C, D). Acute treatment of MI myocytes with NAC (MI-NACa) partially restored the transmural gradient of contractility by increasing SL shortening and the relaxation velocity only in the Endo layer (Fig. 6B-D). To verify that the beneficial effects observed with NAC in $\mathrm{MI}$ rats were not specific to this antioxidant, we tested other antioxidants with a different mode of action, such as Vitamin $\mathrm{C}$, a ROS scanvenger with nonenzymatic mechanism, and $\mathrm{Mn}(\mathrm{III})$ tetrakis (4-benzoic acid) porphyrin (MnTBAP), a cellpermeable SOD mimetic. Both antioxidants restored completely the amplitude of shortening in Endo MI myocytes and improved also the shortening and relaxation velocities, but values remained still lower than sham animals (Supplementary Fig. S3). Chronic treatment of MI rats with NAC (MINACc) fully restored all contractile parameters in Endo cells to sham levels and increased SL shortening also in Epi cells. In physiological conditions, rodents express mostly the fast $\alpha$ myosin heavy chain (MHC) isoform, which is characterized by higher ATPase activity, faster shortening velocity, and faster rates of force development than the slow $\beta$-MHC isoform (33), which is re-expressed during HF (34). Therefore, we investigated whether complete recovery of the contractile function after chronic ROS scavenging compared with acute treatment could be due to modification of $\beta$-MHC expression. The expression of $\beta$-MHC increased significantly only in Endo myocytes from MI rats compared with sham and MI-NACc rats (Supplementary Fig. S5). Thus, the lower expression of the $\beta$-MHC protein in MI-NACc rats might participate in the restoration of the contractile kinetics and amplitude of $\mathrm{HF}$ myocytes following chronic NAC treatment.

Finally, we evaluated the effect of antioxidant treatment on the in vivo contractile properties of MI rats that had been chronically treated with NAC. NAC had minor effects on the cardiac remodeling induced by MI with a modest, but significant reduction of LV dilatation (Table 1). In vivo, the contractile function, investigated by Doppler echocardiography, was largely deficient in MI animals as indicated by their lower endsystolic strain $(-72 \%)$ and aortic velocity time integral $(-38 \%)$ in comparison to sham animals (Fig. 7B, C). Chronic NAC treatment improved the end-systolic strain $(-25 \%$ vs. sham) and restored aortic velocity time integral (Fig. 7C). Moreover, the ratio between the transmitral peaks of the early $(\mathrm{E})$ and late (A) diastolic velocities, which was significantly increased by $168 \%$ in the MI group, was restored to sham levels following NAC treatment (Fig. 7D and Table 1). NAC treatment also restored the transmural gradient of shortening velocity (Vendo-Vepi) that was abolished in MI animals, suggesting a regional improvement of contractility (Fig. 7E and Table 1). 
A
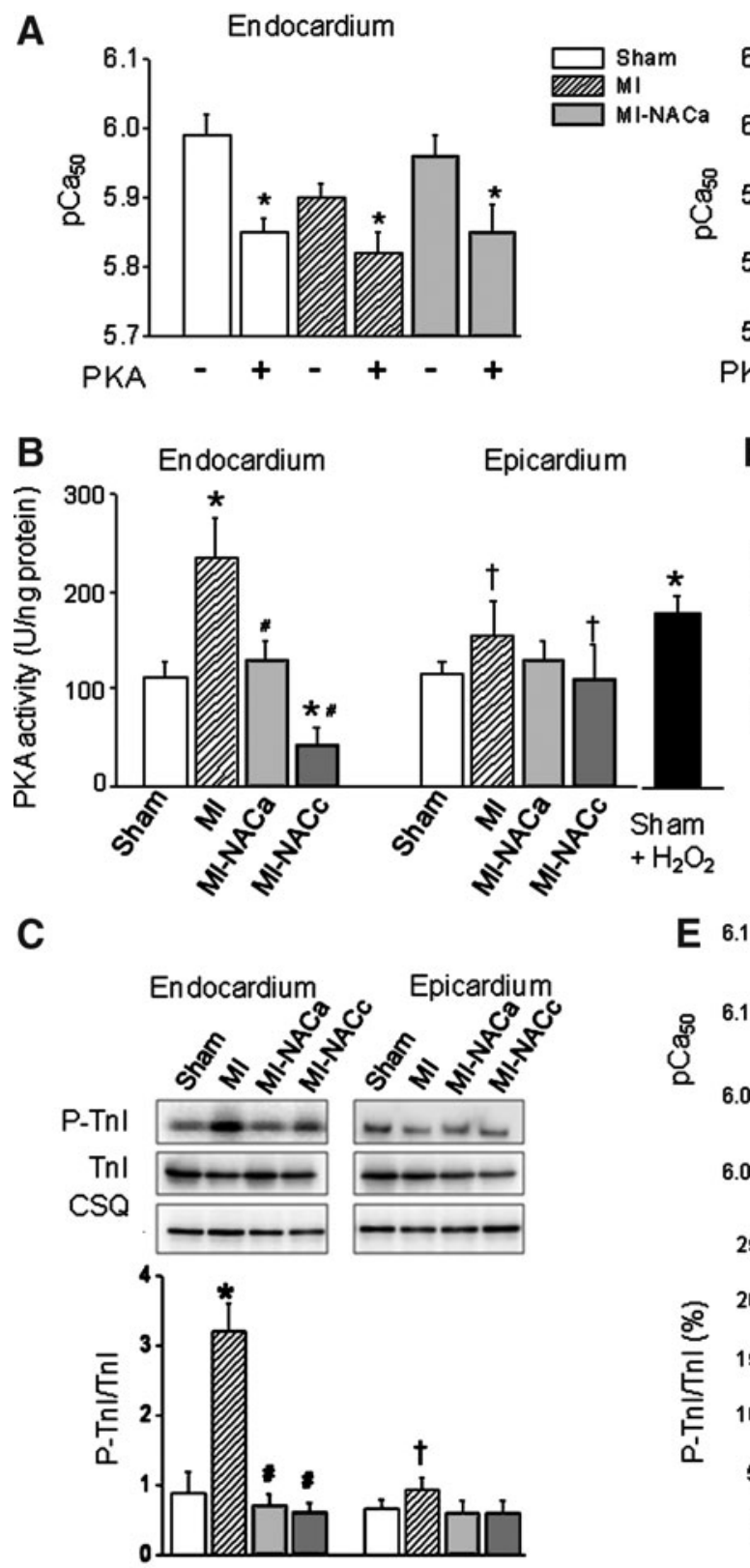

Epicardium

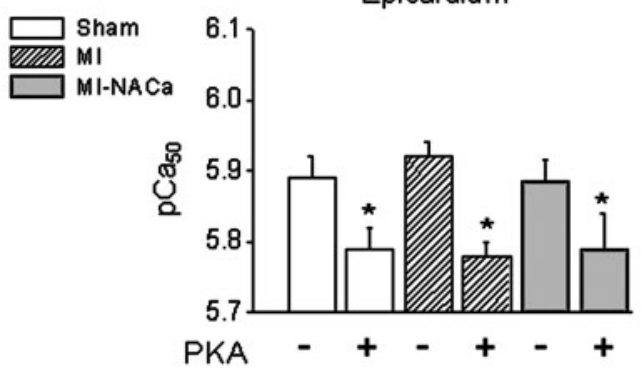

D Endocardium Epicardium

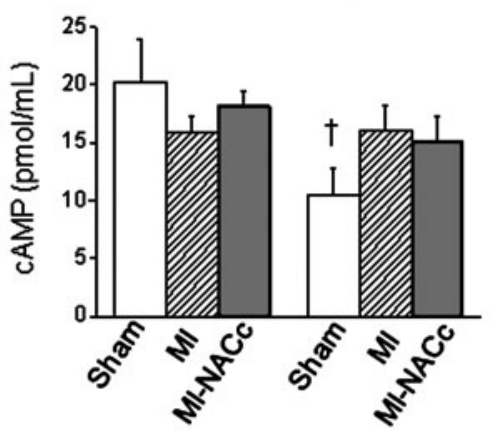

E 6.15

15

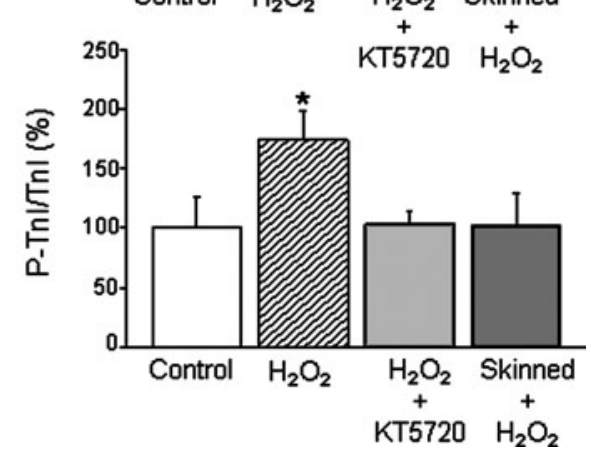

FIG. 5. ROS-induced PKA activation and PKA-dependent phosphorylation of cTnI. (A) Myofilament Ca ${ }^{2+}$ sensitivity was evaluated without and after PKA treatment $(100 \mathrm{U} / \mathrm{ml}$, incubation for $50 \mathrm{~min})$ in permeabilized myocytes from sham, MI, and MI-NACa rats $\left({ }^{*} p<0.05\right.$ PKA vs. without PKA). (B) PKA activity measured in Endo and Epi strips isolated from sham, MI, and MI-NACa or MI-NACc rats $(n=8-10$ strips/4 hearts). The black bar represents the PKA activity of sham myocytes preincubated with $0.1 \mathrm{mM} \mathrm{H}_{2} \mathrm{O}_{2}$ for $15 \mathrm{~min}$. (C) Effect of acute and chronic NAC treatment on PKA-dependent phosphorylation of cTnI in Endo and Epi strips. Phosphorylation levels were determined by Western blotting with an antibody against cTnI phosphorylated at the PKA sites $\operatorname{Ser}^{22} / \operatorname{Ser}^{23}$ (P-cTnI). ${ }^{*} p<0.05$ MI versus sham, ${ }^{\#} p<0.05$ MI-NAC versus MI, ${ }^{\dagger} p<0.05$ Endo versus Epi. (D) cAMP concentration in isolated cardiomyocytes ( $n=5$ hearts $/$ condition). ${ }^{\dagger} p<0.05$ Endo versus. Epi. (E) Myofilament $\mathrm{Ca}^{2+}$ sensitivity (upper panel) and $\mathrm{cTnI}$ phosphorylation level (lower panel) in sham myocytes stimulated or not (control) with $0.1 \mathrm{mM} \mathrm{H}_{2} \mathrm{O}_{2}$ for $15 \mathrm{~min}$. Intact myocytes were incubated with $\mathrm{H}_{2} \mathrm{O}_{2}$ in the presence or not of a specific permeant PKA inhibitor ( $2 \mu M$ KT5720) before permeabilization. Skinned myocytes were also stimulated with $\mathrm{H}_{2} \mathrm{O}_{2}$ (dark gray column) $(n=12-16$ cells $/ 4$ hearts). The phosphorylation level of cTnI is expressed relative to the level of nonstimulated cells. ${ }^{*} p<0.05$ versus control. PKA, protein kinase A. 

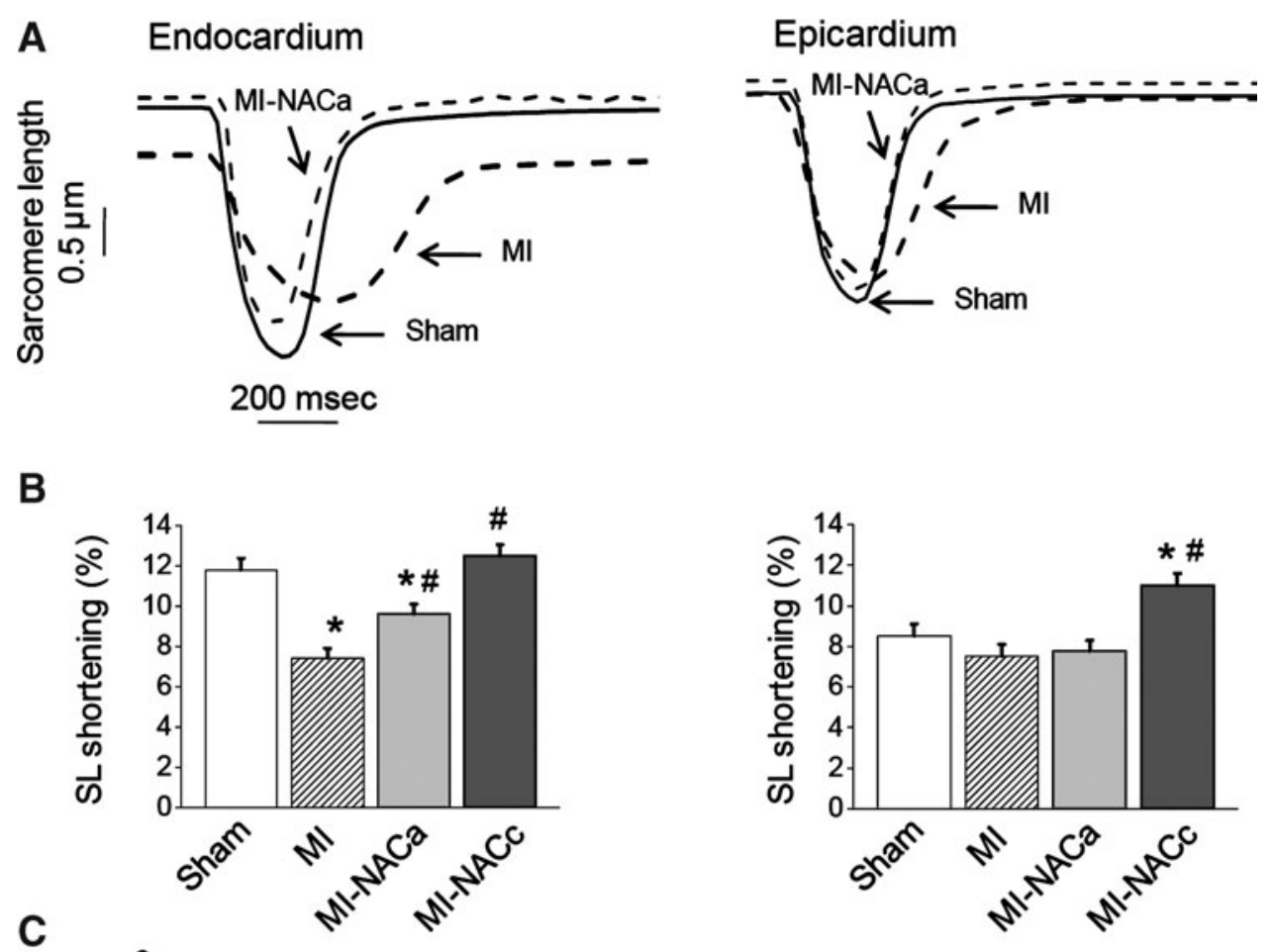

FIG. 6. Regionalized ROSdependent contractile dysfunction in MI rats. (A) Representative recordings of cell shortening at $0.5 \mathrm{~Hz}$ in Endo (left panel) and Epi (right panel) myocytes isolated from sham (full line) and MI rats (dark dashed line). Some MI myocytes were pretreated for $1 \mathrm{~h}$ with NAC (MI-NACa, light dashed line). Quantification of sarcomere length shortening (B) and contraction $(C)$ and relaxation velocities (D) of sham (open bars), MI (dashed bars), and MI-NACa (gray filled bars) or MI-NACc (dark gray filled bars) cells from both Endo and Epi layers (35-50 cells/4-5 hearts). ${ }^{*} p<0.05$ versus sham, ${ }^{\#} p<0.05$ versus MI.
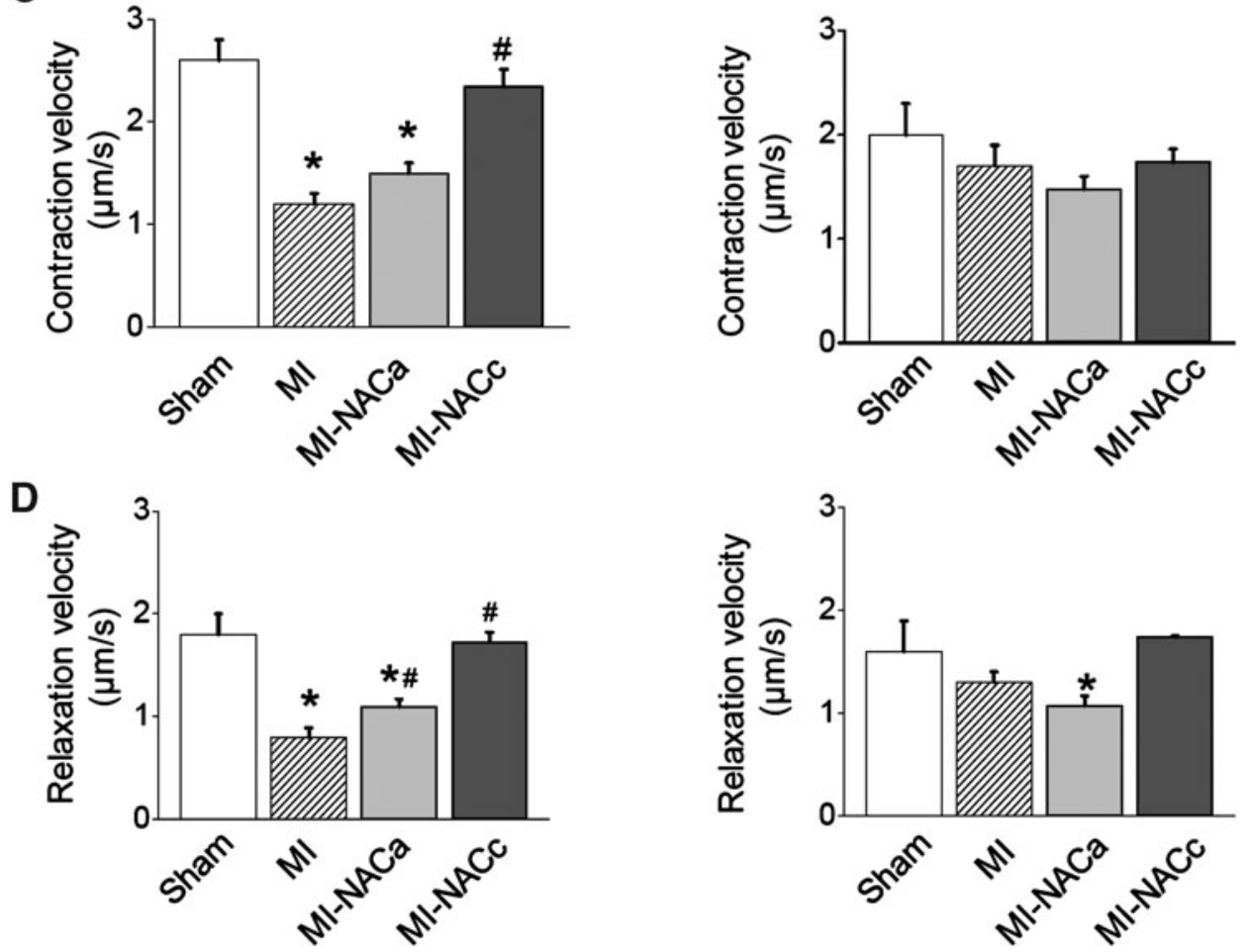

\section{Discussion}

This study highlights the impact of a regionalized mitochondrial ROS production imbalance on myofilament contractile properties in HF. We show that 12 weeks after MI: (i) the transmural contractile gradient has disappeared and this change is associated with a regionalized increased of ROS production in sub-Endo myocytes; (ii) regionalized oxidative stress affects myofilament function in sub-Endo myocytes, at least, in part, through a PKA-dependent pathway; (iii) antioxidant treatment prevents ROS over-production and im- proves the cardiac function in vivo, restores the transmural gradient of contractility across the left ventricular free wall and improves the cellular cardiac function.

\section{ROS promote PKA-dependent suppression of the transmural contractile gradient}

The present study shows that myocytes isolated from the sub-Endo of failing hearts produce more ROS than sham myocytes during a similar sequence of contraction, which affects the properties of their contractile machinery. This was 
ANDRE ET AL.

Table 1. Cardiac Morphological and Functional Parameters in Sham and 12-Week Post-Mi Rats That Have Been Treated (MI-NACc) or Not (MI) with NAC for 4 Weeks

\begin{tabular}{lccc}
\hline & Sham & MI & MI-NACc \\
\hline Body weight (g) & $454 \pm 9$ & $486 \pm 23$ & $453 \pm 15$ \\
Heart mass (g) & $1.78 \pm 0.12$ & $2.19 \pm 0.13^{\mathrm{a}}$ & $2.17 \pm 0.16^{\mathrm{a}}$ \\
Heart mass/body weight (mg/g) & $3.9 \pm 0.1$ & $4.5 \pm 0.2^{\mathrm{a}}$ & $4.8 \pm 0.3^{\mathrm{a}}$ \\
LVESD (mm) & $5.8 \pm 0.2$ & $11.2 \pm 0.1^{\mathrm{a}}$ & $9.8 \pm 0.2^{\mathrm{a}, \mathrm{b}}$ \\
LVEDD (mm) & $9.7 \pm 0.2$ & $12.7 \pm 0.1^{\mathrm{a}}$ & $12.0 \pm 0.1^{\mathrm{a}, \mathrm{b}}$ \\
PWTd (mm) & $1.25 \pm 0.07$ & $1.28 \pm 0.06$ & $1.44 \pm 0.04$ \\
LV shortening fraction (\%) & $39.5 \pm 0.95$ & $12.1 \pm 0.92^{\mathrm{a}}$ & $18.9 \pm 0.93^{\mathrm{a}, \mathrm{b}}$ \\
HR (bpm) & $295 \pm 8$ & $299 \pm 7$ & $294 \pm 9$ \\
Pulsed-wave Doppler & & & \\
E wave (m/s) & $0.90 \pm 0.05$ & $1.0 \pm 0.03$ & $0.93 \pm 0.04$ \\
A wave (m/s) & $0.49 \pm 0.04$ & $0.21 \pm 0.01^{\mathrm{a}}$ & $0.54 \pm 0.06^{\mathrm{b}}$ \\
E/A ratio & $1.84 \pm 0.06$ & $4.94 \pm 0.21^{\mathrm{a}}$ & $1.81 \pm 0.22^{\mathrm{b}}$ \\
Tissue Doppler imaging & & & \\
Endocardial velocity (mm/s) & $50 \pm 2$ & $28 \pm 1^{\mathrm{a}}$ & $27 \pm 1^{\mathrm{a}}$ \\
Epicardial velocity (mm/s) & $31 \pm 1$ & & $27 \pm 1^{\mathrm{a}}$ \\
\hline
\end{tabular}

Values are the mean \pm SEM $(n=7$ animals per group).

${ }^{a}$ Sham versus MI/MI-NAC.

${ }^{\mathrm{b}}$ MI versus MI-NAC; $p<0.05$.

LVESD, left ventricular end-systolic diameter; LVEDD, left ventricular end-diastolic diameter; PWTd, end-diastolic posterior wall thickness; MI, myocardial infarction; NAC, N-acetylcysteine.

reversed with acute antioxidant treatment with $\mathrm{NAC}$, in part, by modulating the PKA activity in a cAMP-independent manner. A previous study showed that hydrogen peroxide activates type I PKA, which translocates from the cytosol to the myofilaments and membranes in cardiomyocytes in a cAMP-independent manner (11). The authors showed that the oxidative disulfide formation in regulatory subunit of type I PKA is associated with subcellular translocation of PKA from the cytosol to the nuclear and myofilament compartments. The lack of difference in the cAMP content between Sham, MI, and MI-NAC myocytes reported here supports a ROSdependent activation of PKA occurring independently of adenylate cyclase/cAMP stimulation without excluding of ROS-dependent increase of PKA sensitivity for cAMP. In line with these results, we show here that blockade of PKA by KT5720 prevented/delayed the $\mathrm{H}_{2} \mathrm{O}_{2}$ effects on cell and SL shortening without affecting the ROS production and $\mathrm{Ca}^{2+}$ transient inhibitions suggesting an implication of the contractile machinery (Supplementary Fig. S4). Moreover, ROS-dependent PKA activation leads to hyperphosphorylation of cTnI at PKA sites, which is a key regulator of myofilaments and which phosphorylation by PKA decreases myofilament $\mathrm{Ca}^{2+}$ sensitivity. The differences in myofilament $\mathrm{Ca}^{2+}$ sensitivity in sham, MI, and MI-NAC rats disappeared after PKA stimulation, suggesting that ROS-dependent activation of PKA modulates the myofilament response. Our work supports an indirect link between ROS production and alteration in cardiac contractility during HF mediated by PKA. This has been shown here in isolated cardiomyocytes, but also in vivo with the chronic NAC treatment. Cell shortening was improved by acute NAC treatment, even restored by Vitamin $\mathrm{C}$ and a SOD mimetic (MnTBAP), in association with restored myofilament $\mathrm{Ca}^{2+}$ sensitivity and normalized PKA activity. However, some contractile parameters, such as the cellular contraction and relaxation velocities, have been poorly improved by acute antioxidant treatment (Fig. 6 and Supplementary Fig. S3) and were restored only after chronic NAC treatment. The contractile beneficial effects after chronic
NAC treatment may be related to various factors, such as the reported changes in the $\beta$-MHC expression in MI-NACc rats and the downregulation of the $\beta$-adrenergic tone. Indeed, a recent study showed that the sympathetic hyperinnervation observed after infarction was blunted after chronic use of NAC, but not vitamins (31). In addition, there is extensive literature showing that expression of the $\beta$-MHC isoform does not affect myofilament $\mathrm{Ca}^{2+}$ sensitivity and the cell shortening amplitude, but accelerates cell and cross-bridge cycling contraction and relaxation velocities (20). Thus, the lower expression of $\beta$-MHC may explain the restoration of cell contraction and relaxation velocities in Endo MI-NACc myocytes compared with MI myocytes and, secondly, it may participate in the in vivo contractile improvements. Other mechanisms are prone to participate to the beneficial effects of chronic NAC treatment. In a similar model, downregulation of the proinflammatory cytokine tumor necrosis factor-alpha and reduction of the fibrosis were reported, which may contribute to the improved in vivo systolic function (1). Finally, increased production of ROS during HF are known to affect nonmyofibrillar proteins involved in the excitation-contraction coupling and, in particular, the proteins involved in $\mathrm{Ca}^{2+}$ handling, such as the Ryanodine receptor $(41,46)$. Thus, the regionalized ROS production and its prevention by antioxidants may also involve $\mathrm{Ca}^{2+}$ homeostasis and may participate in the alterations on contractility observed in the present study.

Previous studies, mostly in vitro, have shown that sarcomeric proteins can undergo a variety of oxidative modifications, including irreversible carbonylation, tyrosine nitration, reversible thiol oxidation, disulfide bond formation, and glutathionylation (40). In vitro oxidation of myofilament protein sulfhydryl groups leads to decreased myofilament $\mathrm{Ca}^{2+}$ sensitivity (24). During HF, carbonylation of cTnI and Tropomyosin has been recently reported in the explanted human heart with end-stage HF using Oxyblot (12). This contrast with the lack of altered oxyblot signal in a recent publication using a mouse model of HF (5) and the present study in a rat model. This negative result is in line with 
A Sham
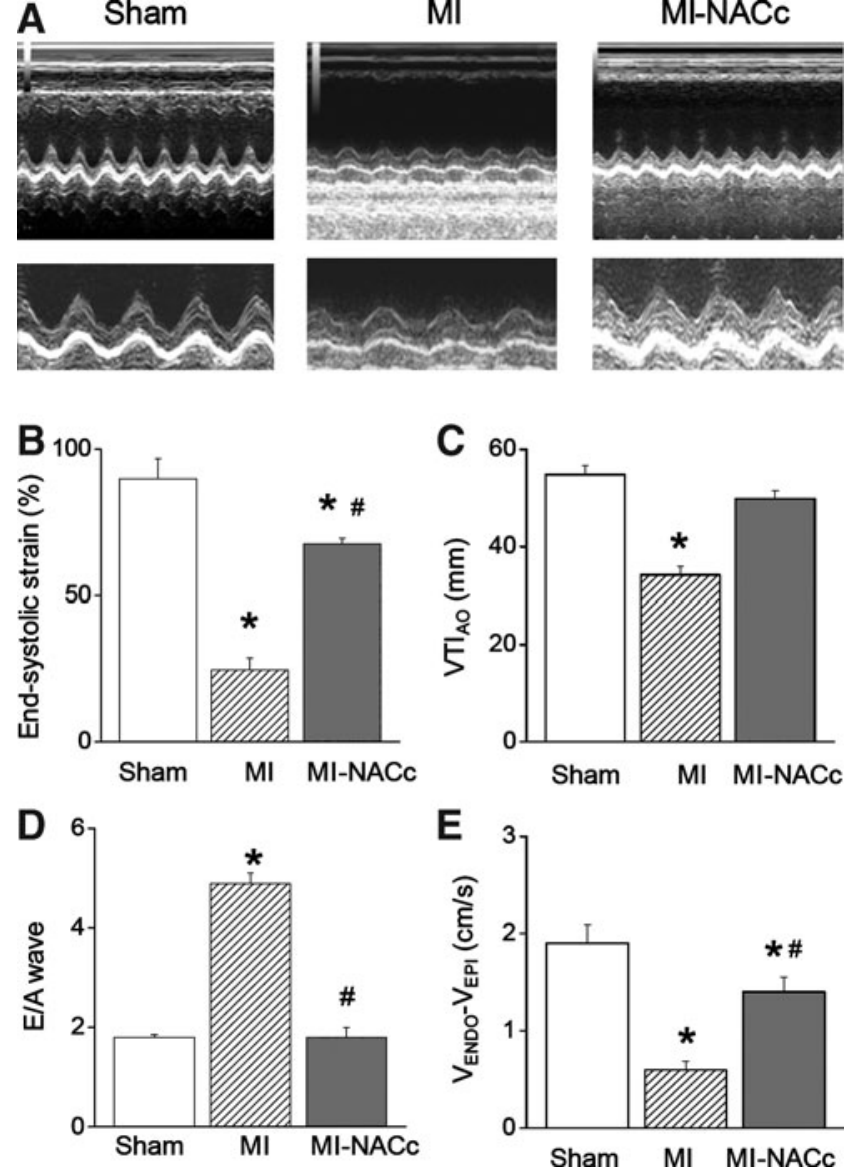

FIG. 7. Effects of chronic NAC treatment (4 weeks) on the cardiac contractile properties. (A) Typical images showing the in vivo cardiac function evaluated by echocardiography. Effects of chronic NAC treatment on end systolic strain (B), aortic velocity time integral $\left(\mathrm{VTI}_{\mathrm{AO}}, \mathrm{C}\right)$, mitral valve $\mathrm{E}$ and $\mathrm{A}$ velocity ratio (E/A) (D), and transmural gradient of velocity (E) in MI (dashed bars), sham (open bars), and MI-NACc (dark bars) rats (seven animals/group). ${ }^{*} p<0.05$ versus sham, $\# p<0.05$ versus MI.

experiments showing the lack of direct effects of $\mathrm{H}_{2} \mathrm{O}_{2}$ on cardiac muscle function (4) and further supports that, in our model, oxidative stress influences indirectly the function of the contractile machinery. As a consequence, contractile proteins are not a major carbonylation target, without excluding other direct oxidative modifications.

The capacity to generate maximal force in MI myocytes was reduced as previously reported $(6,43)$. This alteration was reversed here by acute NAC treatment. The reversibility of the effect excludes cTnI degradation as a mechanism implicated in the reduction of the maximal tension (43), and suggests rather a neurohormonal activation. The decrease of maximal tension in MI rats is classically not attributed to PKA $(18,27)$, but to upregulation of PKC $\alpha$ signaling that causes PKCdependent hyperphosphorylation of myofilament proteins (6). Moreover, $\mathrm{H}_{2} \mathrm{O}_{2}(5 \mathrm{mM})$ has been recently reported to affect myofilament function and to decrease the maximal active tension by indirect post-translational modification of sarcomeric proteins through PKC signaling (4). Thus, ROSdependent activation of PKC during HF in our model could occur and remains to be explored.

\section{Regional mitochondrial dysfunction in HF}

The coupling between metabolic and contractile properties is heterogeneous across $\mathrm{LV}$, since the whole heart efficiency is driven by a transmural contractile gradient. The Endo layer displays a higher myocardium strain rate, circumferential shortening, and velocities compared to the Epi layer (17); thus, it requires a higher metabolism (7) and more oxygen extraction (8). We show here that the higher contractile metabolic demand of Endo myocytes is correlated with increased levels of CS and electron transport chain (ETC) activity in comparison to the Epi layer. However, despite the higher demand of Endo cells, the net production of ROS during contraction is similar in Endo and Epi myocytes from sham animals. This may be explained by the higher antioxidant activities in Endo cells from these animals. During HF, various studies in animal models (42) and in human (37) found either unchanged or decreased antioxidant capacity depending on the enzyme investigated or the stage of the pathology (acute versus chronic ischemia). In the present study, we found regionalized antioxidant capacities reduction in hearts from MI rats despite global cardiac alterations. A possible explanation is that the myocardial blood flow and oxygen delivery are more severely affected in the inner Endo layer than in the outer Epi layer $(25,36)$. This regionalized ischemia may, thus, hinder sufficient oxygen extraction and subsequent NADH oxidation and could explain the potential altered mitochondrial respiratory chain activity in the Endo layer of failing hearts. In addition, mitochondria from sub-Epi and sub-Endo layers of the normal canine myocardium have a similar density, but differ in size and shape (45). Acute MI resulted in the selective loss of a subpopulation of mitochondria prevalent in the subEndo. This would imply greater vulnerability of this layer to anoxia. Impairment of the ETC activity (mainly defects in complex I activity) promotes superoxide anion production (26). The net production of ROS during contraction depends both on the production and scavenging by antioxidant defenses. In Endo MI layer, this mechanism is overwhelmed and can be restored by NAC treatment. The restoration of the contractility in Endo MI myocytes by acute treatment with other antioxidants (vitamin C and MnTBAP, Supplementary Fig. S3) further supports the importance of the antioxidant reserve. To further explore the role of antioxidant capacities on mitochondrial ROS production, we have measured the ROS production in Endo MI myocytes acutely treated with NAC (see Supplementary Fig. S3B). By increasing acutely the antioxidant capacities, the ROS production in Endo MI myocytes was similar to sham Endo myocytes. Those results associated with the regionalized decrease in SOD/catalase activities would be in favor that oxidative stress in Endo MI myocytes is primarily due to the decline in antioxidant defenses rather than the enhancement of mitochondrial pro-oxidant generation.

\section{Clinical relevance}

The transmural gradient of contractility has major contribution for the normal functioning of the heart by coordinating the sub-Endo and sub-Epi layers as they have different temporal activation and level of stretch during a cardiac cycle (9). Here we show that this spatial heterogeneity of cardiac function includes also a spatial heterogeneity of mitochondrial metabolism. Regionalized mitochondrial dysfunction during HF induced localized ROS over production that 
contributed to the homogenization of the contractile function across LV and to the loss of the global contractile gradient. Recovery of the transmural gradient of contraction in HF is, thus, essential for the heart and has been previously observed following a 4-week-moderate exercise program in MI rats with end-stage HF $(14,34)$. Interestingly, exercise training is a physiological way to decrease oxidative stress (39), reduce adrenergic tone, and restore the cellular response to $\beta$-adrenergic stimulation in $\mathrm{HF}$ (32). The beneficial effects of chronic NAC on in vivo and in cellular contractility observed in this study support a link between oxidative stress, activation of PKA, and cardiac contractile dysfunction and, therefore, support the use of antioxidant supplementation as a possible complementary treatment to the current medical therapies, such as $\beta$-blockers, for HF patients.

\section{Materials and Methods}

A detailed Methods section is available in the Supplementary Data.

\section{Animals}

Experiments were carried out in agreement with the Guide for the Care and Use of Laboratory Animals published by the U.S. National Institute of Health (NIH Publications No. 85-23, revised 1996) and with the approval of the French Agriculture Department. Male Wistar rats (5 week/old; Charles River) were randomly subjected either to permanent left coronary artery ligation to induce MI rats or to sham procedure (sham rats) (15). All experiments were performed 12 weeks postligature (Fig. 1). At this stage, MI animals showed signs of cardiac failure, as indicated by the significant reduction of the ejection fraction $(39 \% \pm 1 \%$ vs. $13 \% \pm 1 \%$, sham vs. MI), LV systolic arterial pressure (126 \pm 13 vs. $118 \pm 3 \mathrm{mmHg}), \mathrm{dP} /$ dtmax $(5113 \pm 161$ vs. $4629 \pm 150 \mathrm{mmHg} / \mathrm{s})$, and $\mathrm{dP} / \mathrm{dtmin}$ $(-4494 \pm 245$ vs. $-3777 \pm 105 \mathrm{mmHg} / \mathrm{s})$. The morphological and functional cardiac remodeling was assessed by Doppler echocardiography as previously described (34). Eight weeks after ligature, when most of the morphological remodeling and HF were established, $17 \mathrm{MI}$ rats (MI-NACc) were treated for 4 weeks with NAC (Sigma) administered in their drinking water $(75 \mathrm{mg} /$ day per animal) (10). Each day, the dose of NAC was dissolved in a predetermined daily volume of water that is drunk by a rat (about $40 \mathrm{ml}$ ).

\section{Cell isolation}

Single LV myocytes were isolated by enzymatic digestion as previously described (2). Following enzymatic perfusion, the transmural infarct zone and the border zone were discarded. The remaining sub-Endo layer (Endo) and sub-Epi layer of the remote infarct zone were dissected and mechanically dissociated. The $\mathrm{Ca}^{2+}$ concentration was gradually increased to $1 \mathrm{mM}$. In some experiments, intact MI myocytes were treated acutely with $20 \mathrm{mM}$ NAC for $1 \mathrm{~h}$ (MI-NACa cells) before the experiments. Cell viability after enzymatic isolation was not different between Sham, MI, and MI-NACc hearts (Supplementary Fig. S1).

\section{Mitochondrial ROS production}

Isolated cardiomyocytes were loaded with $5 \mu \mathrm{M}$ MitoSOX Red (Invitrogen) at room temperature for $15 \mathrm{~min}$ followed by washout to evaluate the dynamic mitochondrial $\mathrm{O}_{2}{ }^{\bullet-}$ production (2). Fluorescence images were recorded using a Zeiss LSM 510 inverted confocal microscope. Cells were paced at $0.5 \mathrm{~Hz}$ using two platinum electrodes $(20 \mathrm{~V}, 1 \mathrm{~ms})$. Images were collected at rest and after 5-min stimulation. The resting fluorescence value (obtained after subtracting the background noise measured outside the cell) was used as the reference level.

The MDA content was measured in myocardial tissue isolated hearts paced at $7 \mathrm{~Hz}$ for $15 \mathrm{~min}$ using the Langendorff apparatus as previously described (2).

\section{Force measurement in permeabilized cardiomyocytes}

Isometric force was measured in single cardiomyocytes permeabilized with Triton $\times 100$ (15). The relationship between $\mathrm{Ca}^{2+}$ activated force and internal $\mathrm{Ca}^{2+}$ concentration was measured at 2.3- $\mu \mathrm{m}$ SL. In some experiments, permeabilized cardiomyocytes were incubated with a recombinant catalytic subunit of PKA (Sigma) at room temperature for $50 \mathrm{~min}(13)$.

\section{Protein analysis}

The enzymatic activities of CS, Catalase, total SOD, and complex I and IV activities were measured by spectrophotometry in rat cardiomyocytes as described elsewhere (2).

Proteins were separated on $10 \%$ sodium dodecyl sulfatepolyacrylamide gel electrophoresis gels and blotted onto nitrocellulose membranes (GE Healthcare). Membranes were incubated at $4^{\circ} \mathrm{C}$ with anti-cTnI and -phospho Ser ${ }^{22 / 23-} \mathrm{CTnI}$ (Hytest) antibodies overnight. cTnI levels were expressed relative to the Calsequestrin content (ABR). Immunodetection was carried out using fluorescent secondary antibodies and revealed with the Odyssey Infrared Imaging System (LI-COR Biosciences). The PKA activity was measured in tissue extracts using a nonradioactive PKA activity assay kit (Assay Designs, France) according to the manufacturer's protocol. The cAMP content was measured in pellets of 200,000 myocytes using a direct cAMP ELISA kit (NewEast Biosciences).

Total myocardial protein carbonylation was measured using the Oxyblot Protein Oxidation Detection Kit (Millipore Corporation) according to the manufacturer's protocol in cardiomyocytes and tissue and was normalized to the cTnI content. In some experiments, TnI was immunoprecipitated from heart homogenate $(100 \mu \mathrm{g})$ with anti-TnI antibody in $0.5 \mathrm{ml}$ of a modified RIPA buffer $(50 \mathrm{mM}$ Tris- $\mathrm{HCl}, \mathrm{pH} 7.4$, $0.9 \% \mathrm{NaCl}, 5.0 \mathrm{mM} \mathrm{NaF}, 1.0 \mathrm{mM} \mathrm{Na} 3 \mathrm{VO}_{4}, 0.5 \%$ Triton-X100, and protease inhibitors) for $2 \mathrm{~h}$ at $4^{\circ} \mathrm{C}$. The samples were incubated with protein A sepharose beads (Amersham Pharmacia Biotech) at $4^{\circ} \mathrm{C}$ for $1 \mathrm{~h}$, washed three times with $1.0 \mathrm{ml}$ RIPA, and treated with the Oxyblot kit.

\section{Statistical analysis}

Data were analyzed using one-way or two-way ANOVA. When significant interactions were found, a Bonferroni post hoc test was applied with $p<0.05$ (SigmaStat 3.5). Data are presented as mean \pm SEM.

\section{Acknowledgments}

This work was supported by the "Institut de la Santé et de la Recherche Médicale" (Inserm), "Région Languedoc Roussillon," The Association Française contre les Myopathies and 
the Leducq Foundation CAERUS transatlantic network of excellence. SR, JF, OC, AL are scientists from the Centre National de la Recherche Scientifique. Patrice Bideaux and Sandrine Gayrard are acknowledged for technical assistance.

\section{Author Disclosure Statement}

No competing financial interests exist.

\section{References}

1. Adamy C, Mulder P, Khouzami L, Andrieu-abadie N, Defer N, Candiani G, Pavoine C, Caramelle P, Souktani R, Le Corvoisier P, Perier M, Kirsch M, Damy T, Berdeaux A, Levade T, Thuillez C, Hittinger L, and Pecker F. Neutral sphingomyelinase inhibition participates to the benefits of $\mathrm{N}$-acetylcysteine treatment in post-myocardial infarction failing heart rats. J Mol Cell Cardiol 43: 344-353, 2007.

2. Andre L, Gouzi F, Thireau J, Meyer G, Boissiere J, Delage M, Abdellaoui A, Feillet-Coudray C, Fouret G, Cristol JP, Lacampagne A, Obert P, Reboul C, Fauconnier J, Hayot M, Richard S, and Cazorla O. Carbon monoxide exposure enhances arrhythmia after cardiac stress: involvement of oxidative stress. Basic Res Cardiol 106: 1235-1246, 2011.

3. Anyukhovsky EP, Sosunov EA, and Rosen MR. Regional differences in electrophysiological properties of epicardium, midmyocardium, and endocardium. In vitro and in vivo correlations. Circulation 94: 1981-1988, 1996.

4. Avner BS, Hinken AC, Yuan C, and Solaro RJ. $\mathrm{H}_{2} \mathrm{O}_{2}$ alters rat cardiac sarcomere function and protein phosphorylation through redox signaling. Am J Physiol Heart Circ Physiol 299: H723-H730, 2010.

5. Avner BS, Shioura KM, Scruggs SB, Grachoff M, Geenen DL, Helseth DL, Jr., Farjah M, Goldspink PH, and John Solaro R. Myocardial infarction in mice alters sarcomeric function via post-translational protein modification. Mol Cell Biochem 363: 203-215, 2012.

6. Belin RJ, Sumandea MP, Allen EJ, Schoenfelt K, Wang H, Solaro RJ, and de Tombe PP. Augmented protein kinase C-alpha-induced myofilament protein phosphorylation contributes to myofilament dysfunction in experimental congestive heart failure. Circ Res 101: 195-204, 2007.

7. Beyar R and Sideman S. Left ventricular mechanics related to the local distribution of oxygen demand throughout the wall. Circ Res 58: 664-677, 1986.

8. Bin JP, Le E, Pelberg RA, Coggins MP, Wei K, and Kaul S. Mechanism of inducible regional dysfunction during dipyridamole stress. Circulation 106: 112-117, 2002.

9. Bogaert J and Rademakers FE. Regional nonuniformity of normal adult human left ventricle. Am J Physiol Heart Circ Physiol 280: H610-H620, 2001.

10. Bourraindeloup M, Adamy C, Candiani G, Cailleret M, Bourin MC, Badoual T, Su JB, Adubeiro S, Roudot-Thoraval F, Dubois-Rande JL, Hittinger L, and Pecker F. N-acetylcysteine treatment normalizes serum tumor necrosis factor-alpha level and hinders the progression of cardiac injury in hypertensive rats. Circulation 110: 2003-2009, 2004.

11. Brennan JP, Bardswell SC, Burgoyne JR, Fuller W, Schroder E, Wait R, Begum S, Kentish JC, and Eaton P. Oxidantinduced activation of type I protein kinase A is mediated by RI subunit interprotein disulfide bond formation. J Biol Chem 281: 21827-21836, 2006.

12. Canton M, Menazza S, Sheeran FL, Polverino de Laureto P, Di Lisa F, and Pepe S. Oxidation of myofibrillar proteins in human heart failure. J Am Coll Cardiol 57: 300-309, 2011.
13. Cazorla O, Ait Mou Y, Goret L, Vassort G, Dauzat M, Lacampagne A, Tanguy S, and Obert P. Effects of high-altitude exercise training on contractile function of rat skinned cardiomyocyte. Cardiovasc Res 71: 652-660, 2006.

14. Cazorla $\mathrm{O}$ and Lacampagne A. Regional variation in myofilament length-dependent activation. Pflugers Arch 462: 1528, 2011.

15. Cazorla O, Szilagyi S, Le Guennec JY, Vassort G, and Lacampagne A. Transmural stretch-dependent regulation of contractile properties in rat heart and its alteration after myocardial infarction. FASEB J 19: 88-90, 2005.

16. Derumeaux G, Mulder P, Richard V, Chagraoui A, Nafeh C, Bauer F, Henry JP, and Thuillez C. Tissue Doppler imaging differentiates physiological from pathological pressureoverload left ventricular hypertrophy in rats. Circulation 105: 1602-1608, 2002.

17. Derumeaux G, Ovize M, Loufoua J, Pontier G, Andre-Fouet $X$, and Cribier A. Assessment of nonuniformity of transmural myocardial velocities by color-coded tissue Doppler imaging: characterization of normal, ischemic, and stunned myocardium. Circulation 101: 1390-1395, 2000.

18. Duncker DJ, Boontje NM, Merkus D, Versteilen A, Krysiak J, Mearini G, El-Armouche A, de Beer VJ, Lamers JM, Carrier L, Walker LA, Linke WA, Stienen GJ, and van der Velden J. Prevention of myofilament dysfunction by beta-blocker therapy in postinfarct remodeling. Circ Heart Fail 2: 233-242, 2009.

19. Feigl EO. Coronary physiology. Physiol Rev 63: 1-205, 1983.

20. Fitzsimons DP, Patel JR, and Moss RL. Role of myosin heavy chain composition in kinetics of force development and relaxation in rat myocardium. J Physiol 513: 171-183, 1998.

21. Furukawa T, Myerburg RJ, Furukawa N, Bassett AL, and Kimura S. Differences in transient outward currents of feline endocardial and epicardial myocytes. Circ Res 67: 1287-1291, 1990.

22. Giordano FJ. Oxygen, oxidative stress, hypoxia, and heart failure. J Clin Invest 115: 500-508, 2005.

23. Hamilton KL, Staib JL, Phillips T, Hess A, Lennon SL, and Powers SK. Exercise, antioxidants, and HSP72: protection against myocardial ischemia/reperfusion. Free Radic Biol Med 34: 800-809, 2003.

24. Hertelendi Z, Toth A, Borbely A, Galajda Z, van der Velden J, Stienen GJ, Edes I, and Papp Z. Oxidation of myofilament protein sulfhydryl groups reduces the contractile force and its $\mathrm{Ca} 2+$ sensitivity in human cardiomyocytes. Antioxid Redox Signal 10: 1175-1184, 2008.

25. Hittinger L, Shannon RP, Bishop SP, Gelpi RJ, and Vatner SF. Subendomyocardial exhaustion of blood flow reserve and increased fibrosis in conscious dogs with heart failure. Circ Res 65: 971-980, 1989.

26. Ide T, Tsutsui H, Kinugawa S, Utsumi H, Kang D, Hattori N, Uchida K, Arimura K, Egashira K, and Takeshita A. Mitochondrial electron transport complex I is a potential source of oxygen free radicals in the failing myocardium. Circ Res 85: 357-363, 1999.

27. Janssen PM and de Tombe PP. Protein kinase A does not alter unloaded velocity of sarcomere shortening in skinned rat cardiac trabeculae. Am J Physiol 273: H2415-H2422, 1997.

28. Kainulainen H, Komulainen J, Leinonen A, Rusko H, and Vihko V. Regional differences of substrate oxidation capacity in rat hearts: effects of extra load and endurance training. Basic Res Cardiol 85: 630-639, 1990.

29. Kaumann A, Bartel S, Molenaar P, Sanders L, Burrell K, Vetter D, Hempel P, Karczewski P, and Krause EG. Activation of beta2-adrenergic receptors hastens relaxation and 
mediates phosphorylation of phospholamban, troponin I, and C-protein in ventricular myocardium from patients with terminal heart failure. Circulation 99: 65-72, 1999.

30. Kelley DE, He J, Menshikova EV, and Ritov VB. Dysfunction of mitochondria in human skeletal muscle in type 2 diabetes. Diabetes 51: 2944-2950, 2002.

31. Lee T-M, Lai P-Y, and Chang N-C. Effect of N-acetylcysteine on sympathetic hyperinnervation in post-infarcted rat hearts. Cardiovasc Res 85: 137-146, 2010.

32. Leosco D, Rengo G, Iaccarino G, Golino L, Marchese M, Fortunato F, Zincarelli C, Sanzari E, Ciccarelli M, Galasso G, Altobelli GG, Conti V, Matrone G, Cimini V, Ferrara N, Filippelli A, Koch WJ, and Rengo F. Exercise promotes angiogenesis and improves beta-adrenergic receptor signalling in the postischaemic failing rat heart. Cardiovasc Res 78: 385-394, 2008.

33. Locher MR, Razumova MV, Stelzer JE, Norman HS, Patel JR, and Moss RL. Determination of rate constants for turnover of myosin isoforms in rat myocardium: implications for in vivo contractile kinetics. Am J Physiol Heart Circ Physiol 297: H247-H256, 2009.

34. Mou YA, Reboul C, Andre L, Lacampagne A, and Cazorla O. Late exercise training improves non-uniformity of transmural myocardial function in rats with ischaemic heart failure. Cardiovasc Res 81: 555-564, 2009.

35. Neubauer S. The failing heart-an engine out of fuel. N Engl J Med 356: 1140-1151, 2007.

36. Path G, Robitaille PM, Merkle H, Tristani M, Zhang J, Garwood M, From AH, Bache RJ, and Ugurbil K. Correlation between transmural high energy phosphate levels and myocardial blood flow in the presence of graded coronary stenosis. Circ Res 67: 660-673, 1990.

37. Sam F, Kerstetter DL, Pimental DR, Mulukutla S, Tabaee A, Bristow MR, Colucci WS, and Sawyer DB. Increased reactive oxygen species production and functional alterations in antioxidant enzymes in human failing myocardium. J Cardiac Fail 11: 473-480, 2005.

38. Stanley WC, Recchia FA, and Lopaschuk GD. Myocardial substrate metabolism in the normal and failing heart. Physiol Rev 85: 1093-1129, 2005.

39. Stolen KQ, Kemppainen J, Ukkonen H, Kalliokoski KK, Luotolahti M, Lehikoinen P, Hamalainen H, Salo T, Airaksinen KE, Nuutila P, and Knuuti J. Exercise training improves biventricular oxidative metabolism and left ventricular efficiency in patients with dilated cardiomyopathy. J Am Coll Cardiol 41: 460-467, 2003.

40. Sumandea MP and Steinberg SF. Redox signaling and cardiac sarcomeres. J Biol Chem 286: 9921-9927, 2011.

41. Takimoto E and Kass DA. Role of oxidative stress in cardiac hypertrophy and remodeling. Hypertension 49: 241-248, 2007.

42. Tsutsui H, Kinugawa S, and Matsushima S. Oxidative stress and heart failure. Am J Physiol Heart Circ Physiol 301: H2181H2190, 2011.

43. van der Velden J, Merkus D, Klarenbeek BR, James AT, Boontje NM, Dekkers DH, Stienen GJ, Lamers JM, and Duncker DJ. Alterations in myofilament function contribute to left ventricular dysfunction in pigs early after myocardial infarction. Circ Res 95: e85-e95, 2004.
44. Wang H, Hiatt WR, Barstow TJ, and Brass EP. Relationships between muscle mitochondrial DNA content, mitochondrial enzyme activity and oxidative capacity in man: alterations with disease. Eur J Appl Physiol Occup Physiol 80: 22-27, 1999.

45. Whitty AJ, Dimino MJ, Elfont EA, Hughes GW, and Repeck MW. Transmural mitochondrial differences in myocardium. Recent Adv Stud Cardiac Struct Metab 11: 349-354, 1976.

46. Zima AV and Blatter LA. Redox regulation of cardiac calcium channels and transporters. Cardiovasc Res 71: 310-321, 2006.

Address correspondence to: Dr. Olivier Cazorla U1046, INSERM Université Montpellier 1 CHU Arnaud de Villeneuve Montpellier 34295 France

E-mail: olivier.cazorla@inserm.fr

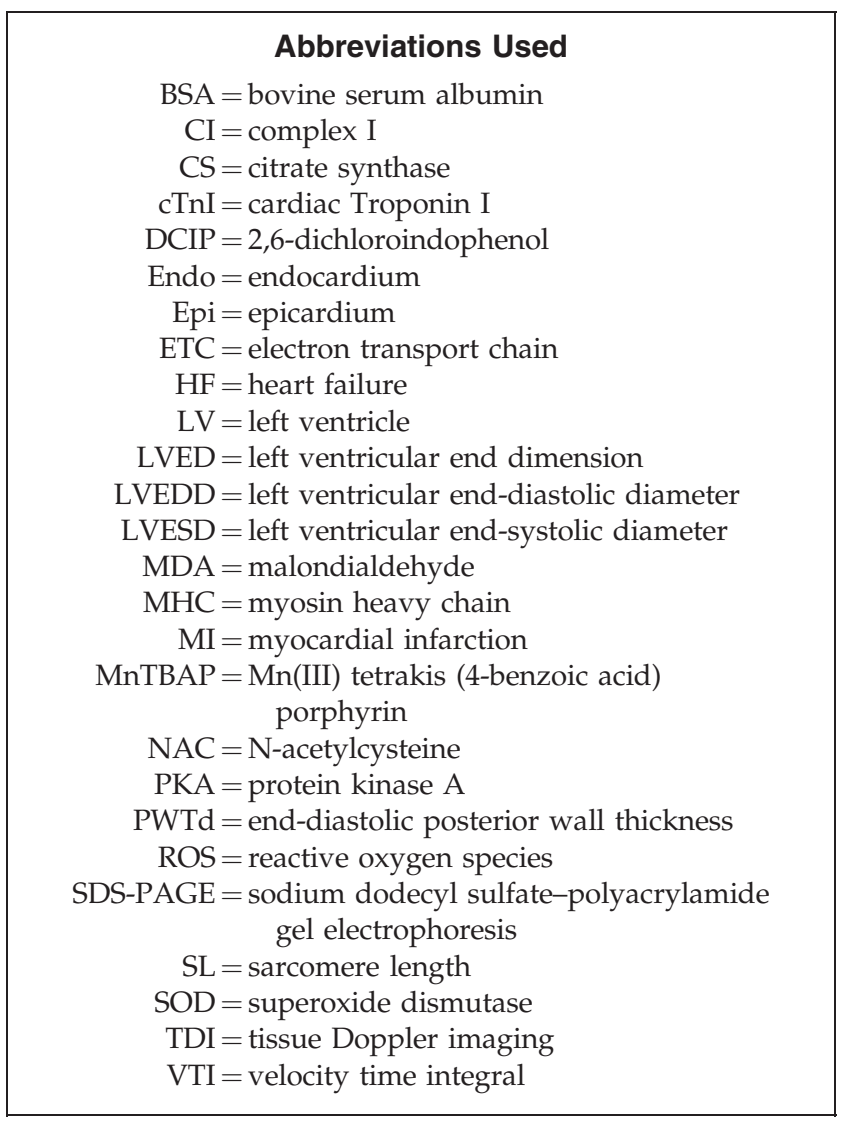

